\title{
The timing of relativistic proton acceleration in the 20 January 2005 flare
}

\author{
G. M. Simnett
}

School of Physics \& Astronomy, University of Birmingham, B15 2TT, UK

e-mail: gms@star.sr.bham.ac.uk

Received 24 May 2005 / Accepted 2 September 2005

\section{ABSTRACT}

Understanding the energy budget in large solar flares requires a good knowledge of how and where the energetic charged particles are accelerated. If they are mainly accelerated by a Coronal Mass Ejection (CME)-driven shock, then they do not have to derive their energy from the flare region. Conversely, if the CME does not accelerate the particles, then the energy must be provided from elsewhere. Resolution of this controversial issue may be aided if we can study events where the timing of the energetic charged particle acceleration may be tightly constrained by the data. We report here on high resolution observations of such an event.

The intense ground level solar proton event of 20 January, 2005 had a rise to maximum at the South Pole of around 5 min, with a similar decay time to $1 / 3$ maximum. This suggests that the magnetic connection from the Sun to the Earth was good and that the proton injection was impulsive on the timescale of a few minutes or less. Comparison of the proton onset time with the solar electromagnetic emissions which accompany large flares, together with observations of the coronal mass ejection seen around the injection time suggests that the CME was not responsible for the relativistic ion acceleration. The near-relativistic $(\sim 250 \mathrm{keV})$ electron intensity onset was some 8 min later than the proton onset. Implications of this on the relative injection time of the particles are discussed. It is concluded that while the relativistic protons were not accelerated by the CME-driven shock, the CME may have influenced the release of both flare-accelerated protons and electrons into the interplanetary medium.

Key words. Sun: flares - Sun: acceleration of particles - Sun: coronal mass ejection (CMEs)

\section{Introduction}

The exact physical processes involved in powering large solar flares remain enigmatic. One of the unknowns is the acceleration of the most energetic particles, namely the protons of energies above $\sim 1 \mathrm{GeV}$. If protons of these energies are produced with a high enough intensity, then on reaching the Earth, a ground level event (GLE) is initiated which may be detected by the network of neutron monitors (NM) around the Earth. In this paper we address the precise timing of relativistic proton acceleration in the flare of 20 January, 2005, relative to other contemporary transient phenomena.

This requires as complete an understanding of particle propagation in interplanetary space as possible, as uncertainties in this respect could lead to errors in interpretation. Particle intensity-time profiles at $1 \mathrm{AU}$ following flares are a mixture of particles propagating directly from the Sun, plus background particles. The latter include remnants from earlier activity, plus flare particles which have propagated along magnetic field lines remote from Earth, which are then backscattered beyond $1 \mathrm{AU}$ to return to the inner heliosphere (Meyer et al. 1956; Bieber et al. 2002). The objective of the analysis is to understand the particle injection profile. The work presented here is also aimed at substantiating our understanding of the propagation.

\section{Statement of the problem}

The acceleration of relativistic protons in flares is a controversial issue. The controversy centres around acceleration by a shock driven by a coronal mass ejection (CME) versus acceleration in the active region. Kahler (1994) studied the injection time-profiles of $>10 \mathrm{MeV}$ protons from 5 flare events associated with fast CMEs and concluded that the peak of the proton injection occurred when the CME was at a height of 5-15 $R_{\odot}$ and that the onset was no earlier than the maximum of the flare impulsive phase. This has been used to support the suggestion of Debrunner et al. (1993) that the relativistic ions are accelerated in a coronal shock rather than in the active region producing the flare.

Resolution of this controversy is long overdue as it has important consequences for the energy balance in flares. If the bulk of the protons are accelerated by the CME-driven shock, then they do not contribute to the flare energy budget, as they would (presumably) derive their energy from the CME, which is on a much larger scale than a flare volume, and may well have more free energy than an active region. On the other hand, if the characteristics of the observed protons are inconsistent with CME-driven shock acceleration then the energy must be 
an integral part of the flare, and be derived from the active region or explained some other way (Simnett 2003).

A comprehensive statistical study of the injection onsets of relativistic protons and electrons was performed by Cliver et al. (1982) who concluded that the order in which the various species were released was $\sim 100 \mathrm{keV}$ electrons, followed within 5 min by the $\sim 2 \mathrm{GeV}$ protons, with the $\sim 1 \mathrm{MeV}$ electrons following at least $5 \mathrm{~min}$ later. For the earliest arriving particles of all species, from a flare favorably located on the Sun at a longitude around $\mathrm{W} 50-60^{\circ}$, scatter-free propagation in the interplanetary medium between the Sun and the Earth is deemed probable (Lockwood et al 1990; Haggerty \& Roelof 2002). Later in the event detection of particles which have been scattered from beyond $1 \mathrm{AU}$ is probable (Meyer et al. 1956; Lockwood et al. 1990; Bieber et al. 2002).

Study of the onsets of solar particle events is made difficult by the fact that the observer at $1 \mathrm{AU}$ will detect particles promptly if they are injected onto field lines connecting to him. In this context the earliest-arriving particles are those which travel without scattering along the magnetic field line connecting the Sun to the Earth, which typically has a length, $L$, of 1.1-1.2 AU. Thus for a particle of velocity $v$, the transit time is $L / v$. The observer will detect the particles with a delay from this time if there has to be cross-field transport near the Sun, if trapping or scattering in the interplanetary medium occurs, or if the particles gradually fill up the inner heliosphere via scattering from a region beyond 1 AU (Meyer et al. 1956). Thus the most definitive results occur when we observe events with a scatter-free onset, which normally would mean the particles arrive as a highly anisotropic beam. This should be borne in mind when comparing different studies.

Recent results from $\sim 100 \mathrm{keV}$ electron studies (Haggerty \& Roelof 2002; Simnett et al. 2002) which concentrated on short, well-connected events with a scatter-free onset (spikes), have shown that there is typically a delay of about 10 min between the electromagnetic signature of a transient event at the Sun and the injection into the interplanetary medium. Haggerty and Roelof found that all the electron events they studied were preceded by a decametric type III radio burst. Most of the events they studied were associated with CMEs, which were typically around 2-3 $R_{\odot}$ (heliocentric distance) when electron injection occurred. However, there is another type of electron event, referred to as a coronal event (Lin 1985; Simnett 2005) where impulsive events are seen at $1 \mathrm{AU}$ which have no clear association with activity in the chromosphere. If coronal events are closely followed by a flare, then the resultant particles at $1 \mathrm{AU}$ will be a mixture of these sources. We suggest here that the $\sim 100 \mathrm{keV}$ electron onsets which Cliver et al. (1982) observed prior to the relativistic proton onsets may come from the "coronal" source.

Kahler et al. (2003) studied the particle onsets for the first ten ground level events seen in solar cyle 23, and compared the onset times of near-relativistic electrons and the protons producing the GLE. They confirmed the Haggerty and Roelof result that the electron injection occurred well after the decametric/hectometric type III radio burst, but found that the four most intense GLE increases had the relativistic proton injection several minutes before the electrons. This suggests that we need to be cautious in interpreting the onset data for weak GLEs, in case there is a threshold/sensitivity effect.

A further consideration is that the onset of a GLE might be caused by relativistic neutrons, which would propagate in a straight line from the Sun to the Earth. Shea et al. (1991) concluded that the onset of the GLE on 24 May, 1990 at groundbased neutron monitors close to the sub-solar point was due to neutrons, and that the proton onset was delayed by around $15 \mathrm{~min}$. The originating flare was located at N36W76 in heliographic coordinates. The arrival of the first relativistic neutrons clearly constrains the acceleration of the primary protons, as the relativistic neutrons have to be produced in a knock-on process from relativistic protons (Ramaty 1986). In the 24 May, 1990 event the first neutrons arrived within a minute of the observed maximum in $\mathrm{H} \alpha$ soft $\mathrm{X}$-rays and $15.4 \mathrm{GHz}$ microwaves. The latter is an indication of relativistic electrons in the chromosphere/low corona. This flare was GOES (Geostationary Operational Environmental Satellites) class X9.3 and had a rise to maximum of only $4 \mathrm{~min}$. Thus the relativistic neutrons were produced within $4 \mathrm{~min}$ of the flare onset. There were no instruments making CME observations at the time of this event.

The timing of the relativistic proton acceleration, both in terms of its duration and its occurrence relative to other signatures of intense flare activity and any associated coronal mass ejection provides important constraints on flare theories. There have recently been detailed analyses of several major ground level events: 14 July 2000 (Bieber et al. 2002), flare longitude W07; 15 April, 2001 (Bieber et al. 2004), flare longitude W85; and 28 October, 2003 (Bieber et al. 2005), flare longitude E08. Of these three events, the risetimes of the NM intensities were $\sim 25 \mathrm{~min}$ (Oulu), $\sim 32 \mathrm{~min}$ (Oulu) and $\sim 30 \mathrm{~min}$ (McMurdo). These results tend to show a complex picture, sometimes involving relativistic solar neutrons (Bieber et al. 2005) and complex proton propagation paths. Note that none of the flares responsible for these events was very close to the nominal magnetic connection longitude of W60, which could explain why the interpretation is complex and why we need to understand the propagation as completely as possible.

Clearly the most definitive data on the acceleration problem comes from the best observed events. An additional constraint comes from solar gamma ray observations, and it was shown by Forrest \& Chupp (1983) that in some flares the electrons and ions were accelerated simultaneously, to within seconds. Thus we have observations of specific events (Shea et al. 1991; Forrest \& Chupp 1983) which show that ion acceleration occurs early in the event, while more statistical studies (Cliver et al. 1982; Kahler 1994) quite naturally would find significant delays, as statistically there would be propagation effects introducing delays.

The role of particle trapping adds a further parameter. One scenario would invoke an erupting magnetic structure (the CME) which may serve to trap any accelerated particles behind it. The higher the erupting structure moves into the corona, the more likely it is to have good access to open magnetic field lines linking to 1 AU. Haggerty \& Roelof (2002) in their study of electron beam event profiles which appeared as intensity spikes lasting 30-60 min, argued against trapping, as this would not produce the uniformity of event profile that they 
observed. Instead, they advocated CME-driven shock acceleration as a likely cause. This received support when Simnett et al. (2002) found an excellent CME association for most of their events. Thus the electron data in spike events strongly support CME-driven shock acceleration. However, the possible effects of trapping cannot simply be dismissed.

One problem that has not been addressed by the CMEshock proponents for the spike events is why the decay from the peak is so rapid. Such a rapid decay suggests an impulsive injection lasting less than $\sim 10 \mathrm{~min}$. The finite risetime provides constraints on propagation delays due to velocity and path length differences. If it is shock acceleration, it would be surprising for this to turn off so rapidly, as a typical fast CME moves through the corona out to $10 \mathrm{~s}$ of $R_{\odot}$ at a constant speed, to the accuracy of the observations. However, the alternative, acceleration matching the timescale of the hard $\mathrm{X}$-ray/microwave pulse, would, after folding in the propagation effects, produce an intensity pulse at $1 \mathrm{AU}$ rather like those observed. For about half the electron spike events there is a long, low level decay where the intensity is isotropic to better than $\sim 10 \%$. This can be measured directly from spacecraft such as the Advanced Composition Explorer (ACE), while it may be inferred from sophisticated analysis of the global network of neutron monitors in some GLE.

The event we discuss in this paper, which occurred on 20 January, 2005, produced protons of energies above $1 \mathrm{GeV}$ with an intensity-time profile having a risetime of $<10 \mathrm{~min}$ with a similar initial decay time, which then developed into a long decay lasting over $2 \mathrm{~h}$. This intensity-time profile was very similar to that of the electron spike events which have been well-studied by Haggerty \& Roelof (2002) and Simnett et al. (2002), where clear limits on the injection time could be obtained. From the benefit of these works we believe we can provide a limit on the injection time for the relativistic protons for the 20 January event. In the following section we present the observations; in Sect. 4 we discuss propagation effects; and in Sect. 5 we address how this event confirms that the relativistic proton acceleration is unlikely to be at the CME-driven shock, but in an earlier process related in time to the X-ray, gamma-ray and microwave emissions.

\section{Observations}

The event we are discussing was a GOES class X 7.1 flare, optical class 2B, from an active region at N12 W58 on the visible solar disc. The onset in $\mathrm{H} \alpha$ was given as 06:41 UT on 20 January, 2005 (Solar Geophysical Data, US Dept. of Commerce, Boulder, $\mathrm{CO}$ ). In the following we shall omit the year unless different from 2005.

The event was well-observed by the "Reuven Ramaty High Energy Solar Spectroscopic Imager" (RHESSI), which made observations from soft X-ray enegies $(3 \mathrm{keV})$ up to gammaray energies $(20 \mathrm{MeV})$. Figure 1 shows some sample light curves from RHESSI. In soft X-rays the event started gradually, and at 06:37 UT there was a short burst which was also seen at 25-50 keV (hard X-rays; HXR). However, the main hard X-ray burst started at 06:38 UT. The 25-50 keV maximum intensity was at 06:46 UT $\pm 1 \mathrm{~min}$, while the maximum in

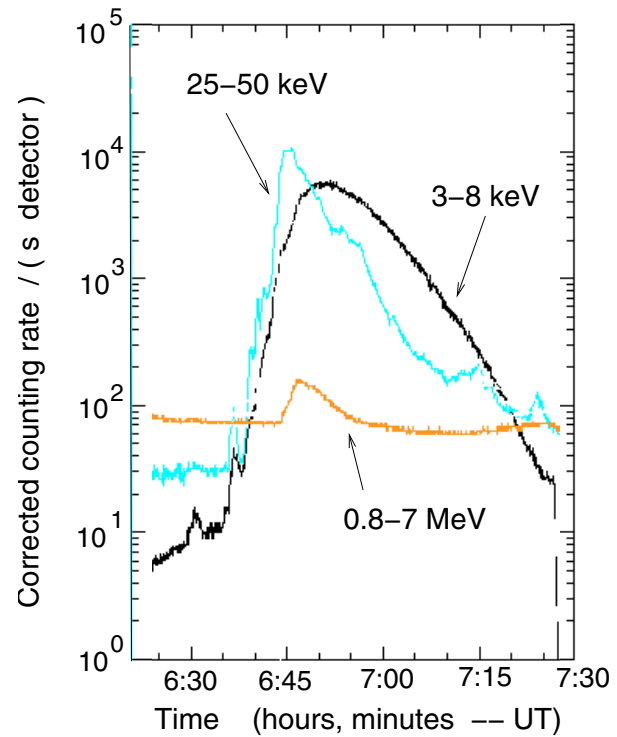

Fig. 1. The $X$ and gamma ray light curves on 20 January, 2005. Black: 3-8 keV, blue: 25-50 keV, orange: $0.8-7 \mathrm{MeV}$ (Data courtesy of the RHESSI team).

the $0.8-7.0 \mathrm{MeV}$ channel, which includes the neutron capture line at $2.223 \mathrm{MeV}$, was at 06:47 UT. The 4-7 MeV gammaray burst, which includes prompt gamma-ray lines of $\mathrm{C}$ and $\mathrm{O}$, in addition to any continuum present, started at 06:44 UT and was a maximum at 06:46 UT (Dr G.H. Share, private communication). An intense microwave burst started at this time also, which reached at least $5.3 \times 10^{8}$ Jansky at $15.4 \mathrm{GHz}$ at 06:44 UT. Although the soft X-ray (SXR) event had a long, low-level decay, the duration (full width at $1 / 10$ maximum) was less than $30 \mathrm{~min}$. Thus this event is an excellent example of an intense, impulsive, very energetic event which released relativistic particles into the interplanetary medium.

\subsection{Energetic particles}

Relativistic protons were detected by the network of neutron monitors around the Earth. The counting rates of the NM operated by the Bartol Research Institute, University of Delaware are shown in Fig. 2 covering the main part of this event (Data courtesy of Professor J.W. Bieber). The event is clearly very anisotropic. The south polar neutron monitor recorded the largest increase, starting no later than 06:49 UT, while other stations such as Thule, Greenland, observed an increase less than $5 \%$ of the South Pole, with the maximum delayed by 37 min. However, other northern latitude monitors showed an impulsive increase similar to the South Pole. The neutron monitor intensity-time profile from Climax (Colorado) is shown in Fig. 3. It had a rise to maximum from the background level, and a decay to $1 / 3$ maximum intensity covering just $7 \mathrm{~min}$, compared to $10 \mathrm{~min}$ for the south polar monitor. Data from other neutron monitors shown in Fig. 2 exhibit a variety of intensitytime profiles which we interpret as due to a combination of anisotropy and cut-off rigidity.

Near-relativistic electrons $(\sim 175-\sim 300 \mathrm{keV})$ from the flare were detected at around 06:57 UT by the Electron, Proton and 


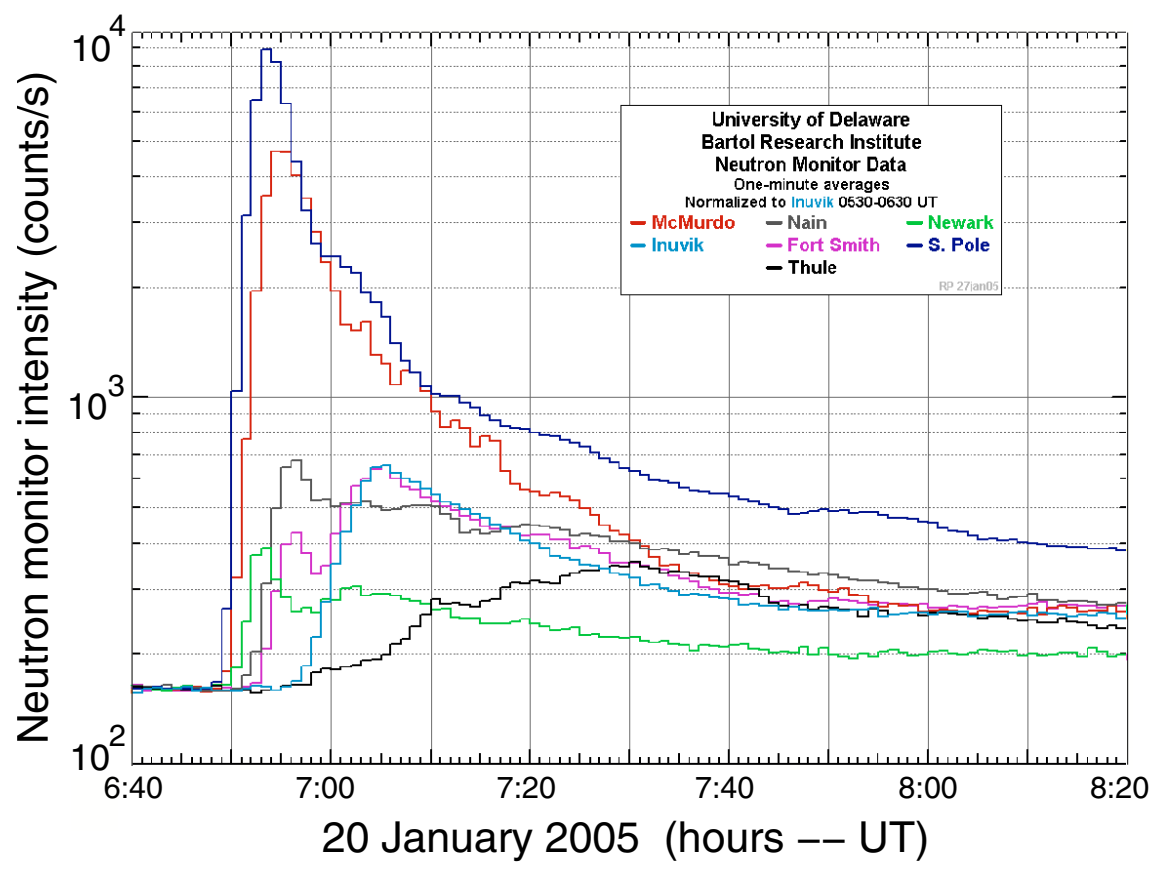

Fig. 2. The counting rate recorded by the neutron monitors from the Bartol Research Institute, University of Delaware on 20 January, 2005.

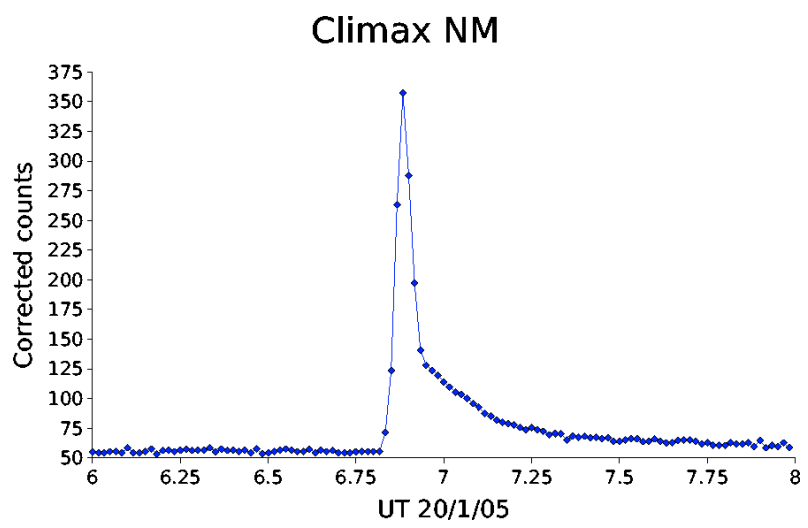

Fig. 3. The counting rate recorded by the Climax (Colorado) neutron monitor on 20 January, 2005. (Data courtesy of Dr C. Lopate)

Alpha Particle Monitor (EPAM) instrument on the ACE spacecraft, which is spin-stabilised at $5 \mathrm{rpm}$. This is $\sim 8 \mathrm{~min}$ after the proton onset. EPAM provides electron data from three separate detectors. These are from the LEFS60 and LEFS150 detectors (see Gold et al. 1998 for a description of the instrument) which are mounted at $60^{\circ}$ and $150^{\circ}$ to the spin axis respectively, and magnetically-deflected electrons, which come through a cone centred at $30^{\circ}$ to the spin axis. All detectors have a full opening angle of approximately $50^{\circ}$. The data are accumulated in either 4 or 8 sectors of the spacecraft spin, so that anisotropy information may be obtained. ACE has a magnetometer (Smith et al. 1998). Figure 4 shows the intensity-time history of the electrons detected above local background in the peak sectors of the three detectors (see figure caption). The detector with both the earliest onset and most rapid rise is LEFS150, sector 4.

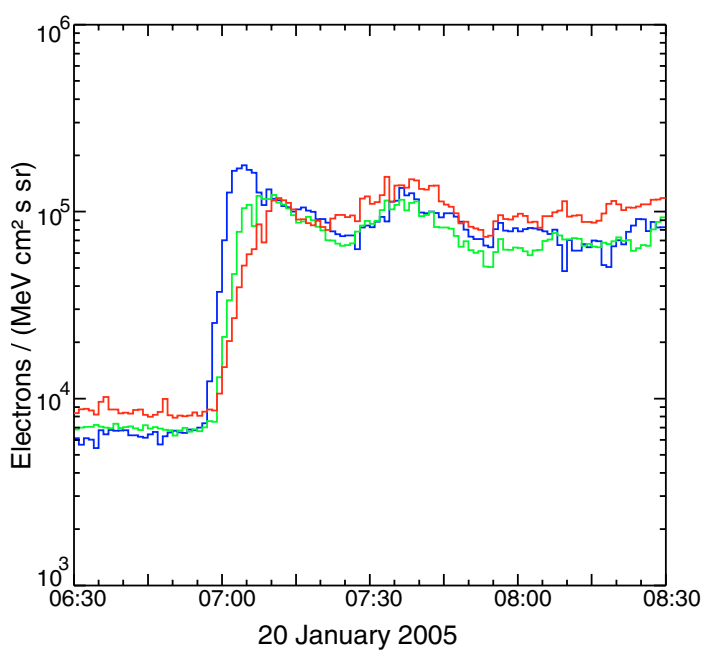

Fig. 4. The $\sim 175-\sim 300 \mathrm{keV}$ electron intensity for the peak sector in the LEFS60 (green, sector 3), LEFS150 (blue, sector 4) and the deflected electrons (red, sector 2) telescopes from 06:30-08:30 UT on 20 January. The data are plotted at a time resolution of $1 \mathrm{~min}$.

\section{2. $\mathrm{SOHO}$ images}

Coronal images provide important information of the progress of the event through the upper solar atmosphere into the interplanetary medium. The imaging observations we use were taken with the Extreme Ultraviolet Imaging Telescope (EIT; Delaboudinière et al. 1995) and the Large Angle Spectroscopic Coronagraph (LASCO; Brueckner et al. 1995) on the Solar and Heliospheric Observatory (SOHO) spacecraft. These images are used to give timing information of moving structures in the low corona (EIT) and CMEs (LASCO). In Fig. 5 we show some images from EIT up to and including the flare onset. The northwest quadrant of the Sun is shown in the upper three images, 


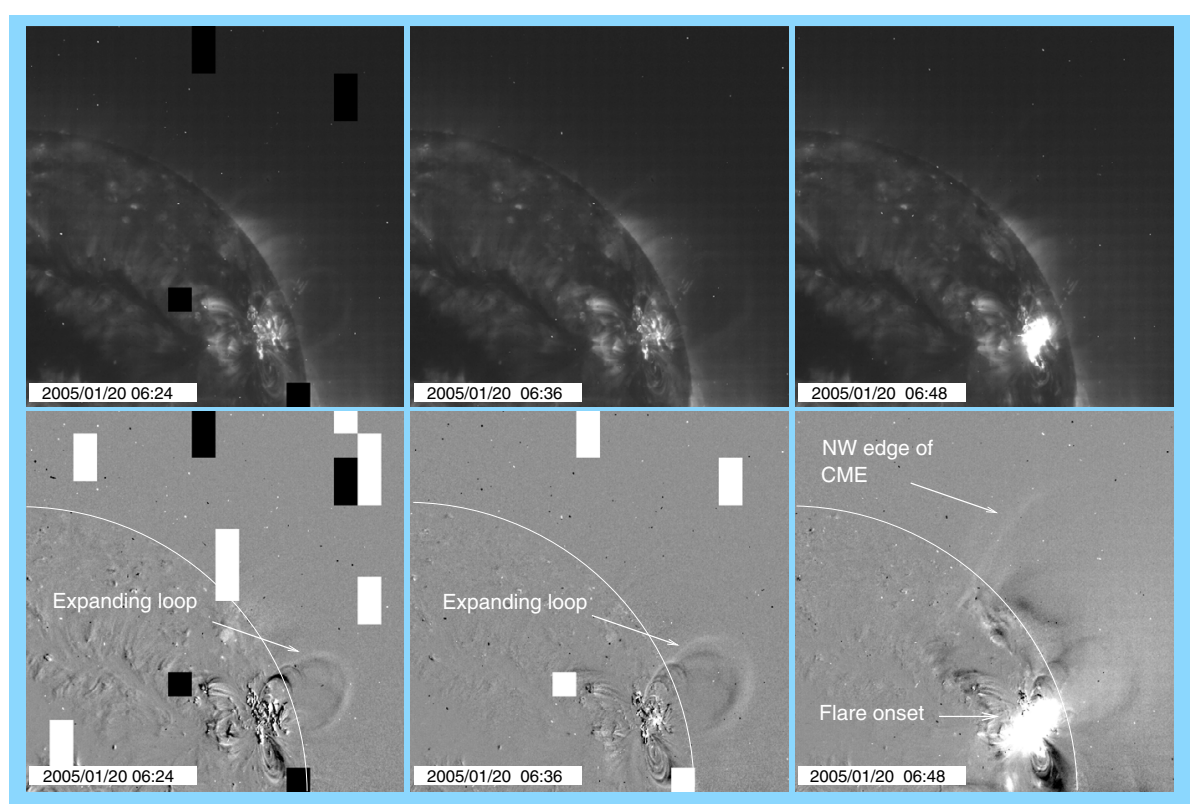

Fig. 5. The eruption of a coronal loop as seen by EIT. The images show the NW quadrant of the Sun. The upper three images cover the active region and show that the onset of the flare is between 06:36 and 06:48 UT. The lower three images are running differences, where the image shown has had the previous EIT image subtracted from it. There is a faint expanding coronal loop visible in the images at 06:24 and 06:36 UT. This has erupted by 06:48 UT. Note in the 06:48 UT image there is an enhancement towards the north, indicated by an arrow, which is consistent with the northwest edge of the CME shown in Fig. 4. The white/black rectangles correspond to missing portions of the images. The position of the solar limb is indicated by the white arc.

which are taken through the $19.5 \mathrm{~nm}$ filter. During active times this has FeXII emission as the dominant contributor. The lower three images are running differences, where the displayed image has had the previous image subtracted from it. The typical cadence for EIT images is $12 \mathrm{~min}$. In the running difference image at 06:24 UT a faint coronal loop is visible. This is slowly expanding upwards into the corona at an estimated speed of $58 \pm 6 \mathrm{~km} \mathrm{~s}^{-1}$ in the plane of the sky, based on the first two images shown in Fig. 5. By the time of the image at 06:48 UT, just after the hard X-ray maximum, this loop has erupted, together with a somewhat more extensive coronal mass ejection, the northwest edge of which is indicated by an arrow in Fig. 5.

The LASCO image from the $\mathrm{C} 2$ coronagraph, taken at 06:54 UT, is shown in Fig. 6. The solar limb is indicated by the white circle, and the occulting disc, which blocks direct solar radiation out to a height of around $2.2 R_{\odot}$, is shown in solid black. Several dotted lines are added to this figure to aid the eye. The two most extreme represent the edges of the CME, and the one nearest the north pole is the edge indicated on the EIT image in Fig. 5. The small white disc represents the flare site and the radial arrow passing through this point represents the top of the expanding (erupting) loop indicated in Fig. 5. Although there is only one useful image of this mass ejection off the west limb (subsequent images are grossly contaminated by charged particles hitting the LASCO CCDs), it is possible that there is more than one erupting structure visible in Fig. 6. Note that the pre-existing streamer is not affected by the transient event. The background noise is partly due to charged particles from the flare hitting the CCD camera.

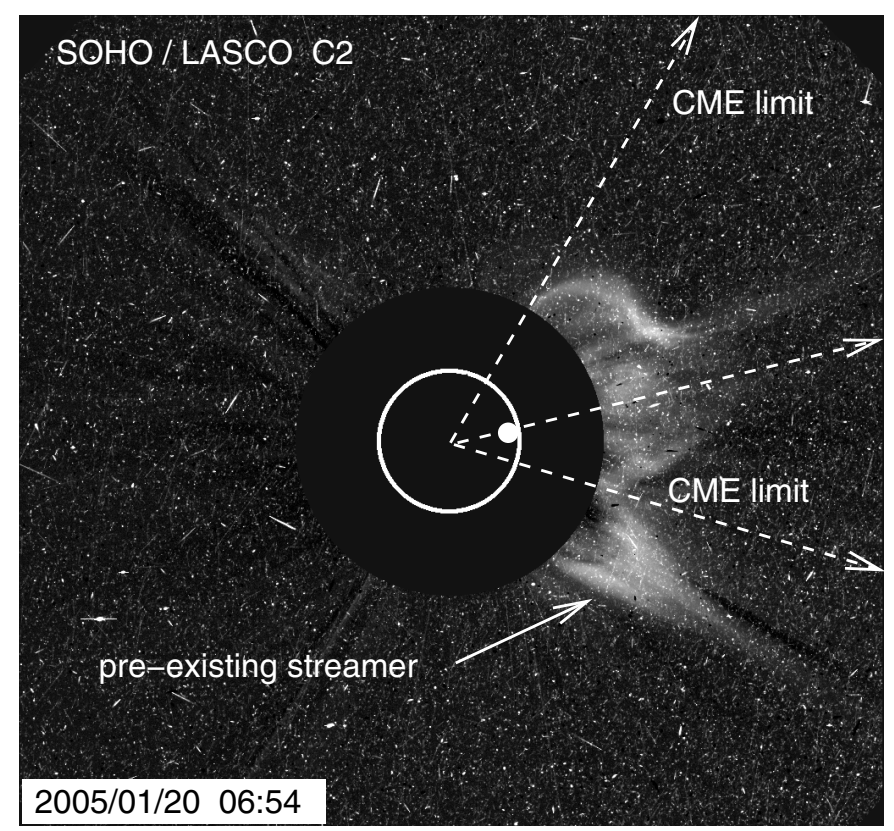

Fig. 6. The image from the LASCO C2 coronagraph at 06:54 UT on 20 January. The outermost dotted lines are the suggested angular limits to the CME, projected back to the centre of the Sun. The white circle represents the photosphere and the black disc represents the position of the $\mathrm{C} 2$ occulter at around $2.2 R_{\odot}$. The solid white circle is at the position of the active region from which the flare erupted and the middle dashed line is a radial line through this position.

\section{Discussion of the particle intensity profiles}

We know from the RHESSI and ground-based solar flare data when the energetic electromagnetic emissions were produced 


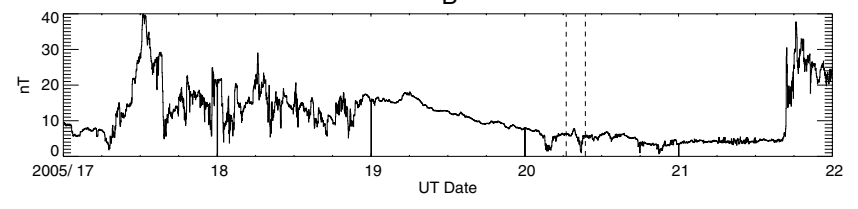

theta

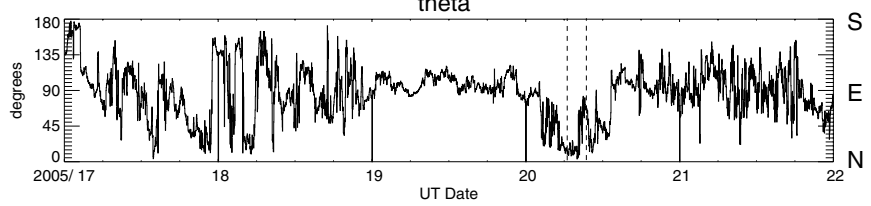

phi

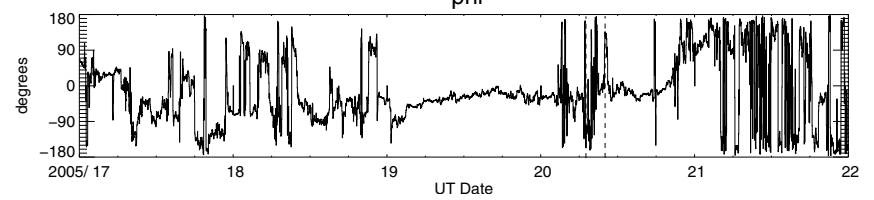

Fig. 7. The magnetic field at ACE from 17-22 January. From the top: the magnetic field intensity (B); the $\theta$ component; the $\phi$ component. North $(\mathrm{N})$, south $(\mathrm{S})$ and ecliptic plane (E) are indicated on the right of the middle panel. The data are plotted as two minute averages. The $\mathrm{B}$ and $\theta$ plots have an artefact at midnight each day. The dashed lines delineate the interval plotted in Fig. 8.

at the Sun. EIT and LASCO data give us timing information on the erupting structures. The charged particle data tell us when the relativistic particles arrived at the Earth. Our task is to coordinate these data to provide timing constraints on the acceleration of the particles at the Sun. However, first we must try to understand the conditions in the interplanetary medium which are influencing or controlling the evolution of the charged particle intensities.

\subsection{Interplanetary propagation}

To obtain unambiguous information on the injection of energetic particles at the Sun, we need to examine the interplanetary magnetic field. The magnetic field at ACE experienced an enhancement to around $40 \mathrm{nT}$ on 17 January and for the next three days dropped spasmodically to the typical field of $\sim 5 \mathrm{nT}$. Figure 7 shows the field magnitude, $B$, and the $\theta, \phi$ components from 17-21 January, plotted in RTN coordinates. RTN coordinates have $\mathrm{R}$ as the outward radial component, $\mathrm{T}$ as the component perpendicular to $\mathrm{R}$ in the ecliptic plane, and $\mathrm{N}$ completes the orthogonal set. $\phi$ is the angle in the ecliptic plane, with the $0^{\circ}$ origin in the outward radial direction, increasing in the anticlockwise direction when viewed from the north. $\theta$ is the angle the magnetic field vector makes out of the ecliptic plane, with $0^{\circ}$ being north and $180^{\circ}$ south. From 19 January onwards the fluctuations which are present for the previous $36 \mathrm{~h}$ have largely died away. In Fig. 8 we show the same field components plotted as 12s spin averages for the period 06-08 UT on 20 January. $\theta$ is typically within $15^{\circ}$ of north for an hour before the onset of the proton event. The $\phi$ component is $315^{\circ}\left(-45^{\circ}\right.$ in Fig. 7) for the nominal Parker field direction if the field is outwards from the Sun.

The 20 January flare occurred during a solar active period, when the background energetic electron intensity near ACE
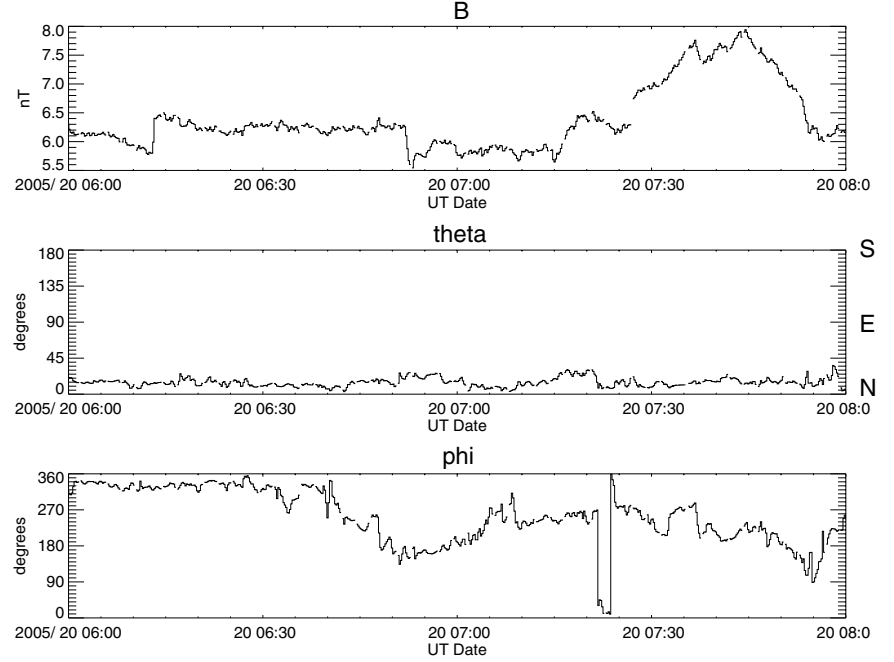

Fig. 8. The magnetic field at ACE from 06-08 UT on 20 January. (As Fig. 7, except at $12 \mathrm{~s}$ resolution) Note that the scale for phi is centred at $180^{\circ}$ to minimise the wrap-around effect.

was enhanced some three orders of magnitude above the nominal background. Therefore we must take this into account. The previous major particle event was on 17 January, associated with a GOES class X 3.8 flare at $\sim 09: 30$ UT, from the same active region as the event we are discussing here. Thus by 20 January the electron intensity near ACE was approximately both constant and isotropic. Therefore we are able to separate the direct electrons from the 20 January flare from this local background intensity to derive an onset time.

It is believed that the inner heliosphere frequently acts as a particle reservoir (Roelof et al. 1992). The outer boundary is thought to be a fast/slow stream interface, after a concept first discussed by Meyer et al. (1956). This could be a corotating interaction region, or alternatively it could be a recent CME. The exact form is not important to the present study. Thus on 20 January the picture we for the electrons is a fresh injection into a volume already populated with a slowly-decaying intensity from the earlier event, plus a magnetically complex pattern in the interplanetary medium beyond $1 \mathrm{AU}$ due to the earlier activity (see Fig. 7).

Now that we have justified the subtraction of a significant background, we are in a position to examine the behaviour of the fresh injection on 20 January. The bulk of the electrons propagate along the magnetic field outwards from the Sun, as may be seen in Fig. 9. The upper panel shows the pitch angle distribution from the LEFS60 detector from 07:06-07:09 UT when the intensity is within a factor of three of the peak intensity seen in the event. It is difficult to measure the onsets to an accuracy of better than 1 min or so, due to the high background from the earlier event. However, the detector which shows the fastest rise is LEFS150, where the intensity (E4) exceeds $10^{4}\left(\mathrm{MeV} \mathrm{cm}^{2} \mathrm{sr} \mathrm{s}\right)^{-1}$ some two minutes before this level is exceeded in either of the other detectors. This is consistent with the magnetic field direction. The lower panel of Fig. 9 shows the pitch angle distribution of the 170-280 keV electrons from 06:58-06:59 UT, as they are rising towards 


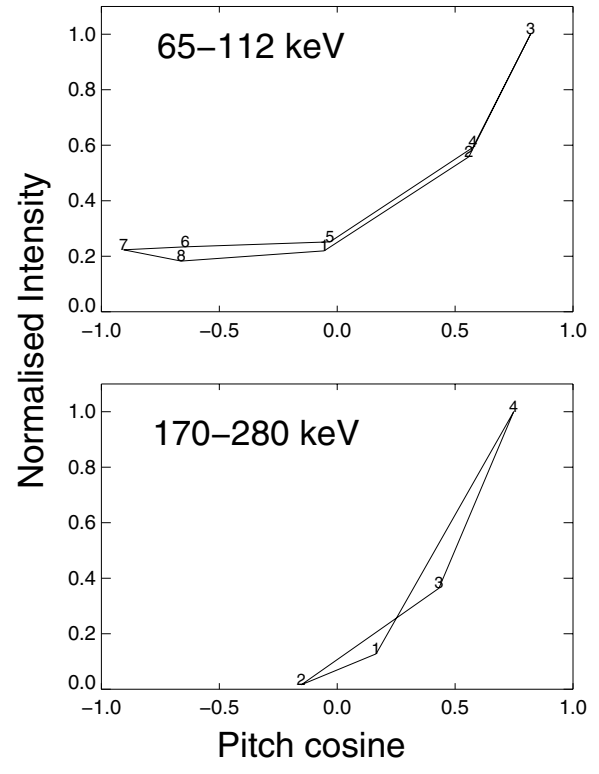

Fig. 9. Upper panel: the $65-112 \mathrm{keV}$ electron pitch angle distribution from the LEFS60 detector from 07:06-07:09 UT on 20 January. The pre-event background of 980 counts/s has been subtracted from all sectors. Lower panel: the $170-280 \mathrm{keV}$ electron pitch angle distribution from the LEFS150 detector from 06:58-06:59 UT on 20 January. The pre-event background level of 250 counts/s has been subtracted from all sectors. The numbers on the plot refer to the spin sector of the accumulation at ACE.

maximum intensity. These distributions demonstrate that initially the electron onset at ACE was a field-aligned beam.

\subsection{Effects of trapping}

The relativistic proton intensity and anisotropy are inferred from the secondary effects caused by their interaction in the Earth's atmosphere. We have no evidence that relativistic protons were produced from the 17 January event and therefore we assume there is a negligible residual background. The neutron monitor intensities shown in Fig. 2 suggest that the proton onset was highly anisotropic, with the highest intensity observed near the sub-solar point in Antarctica. The neutron monitor at Inuvik is at a high geomagnetic latitude $\left(\sim 71^{\circ} \mathrm{N}\right)$ with a cut-off rigidity of only $0.17 \mathrm{GV}$ (Shea et al. 1991), and yet its response was not only delayed from that at the South Pole, but the maximum was $\ll 10 \%$ of that station. However, during the decay the intensity at most of the neutron monitors became almost equal, to better than a factor of two, as may be seen from Fig. 2. This we attribute to the reservoir concept discussed above, with a scattering boundary somewhat beyond the orbit of Earth. However, the exception is that at the South Pole itself. This station also has a low cutoff rigidity and high sensitivity due to its altitude of $2820 \mathrm{~m}$. Therefore this suggests continued emission from the Sun at a decreasing level of a few $\%$ of the peak intensity. We advocate trapping of protons behind the CME front, with slow leakage, to account for the continued anisotropy, and trapping within the inner heliosphere to account for the long decay.

Trapping was advocated by Bieber et al. (2005) to explain the long decay of the GLE following the 28 October 2003 flare,

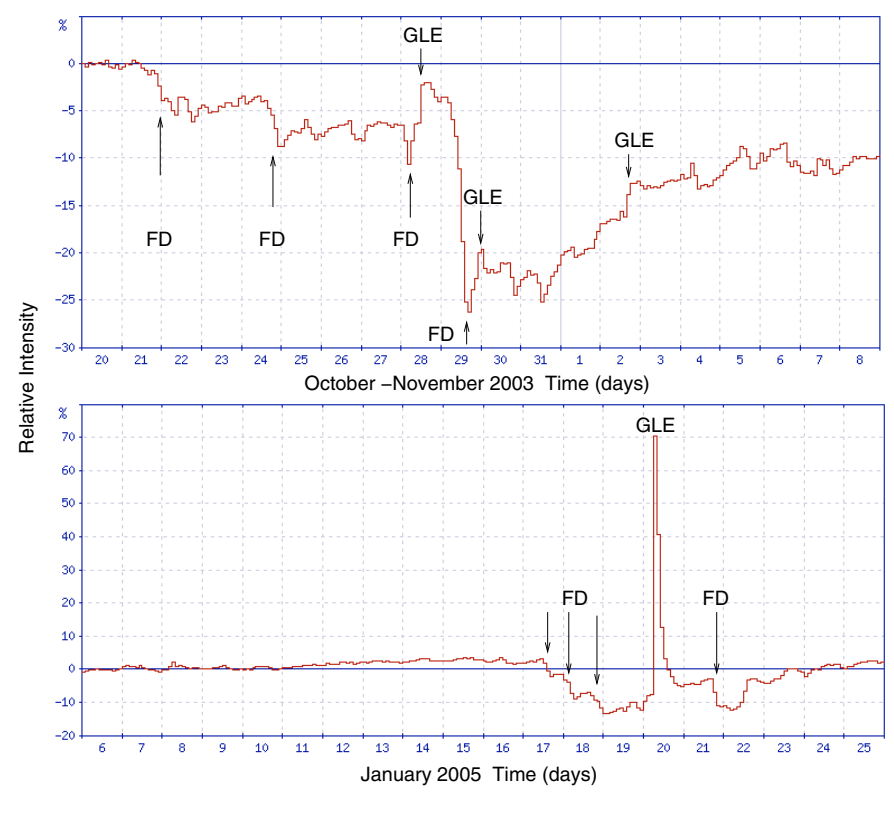

Fig. 10. The neutron monitor records for 20 days in October/November, 2003 and January, 2005 from Oulu, Finland. (Data courtesy of Dr Ilya Usoskin).

which also had an intensity spike at the onset, albeit of much longer duration than the event we are discussing here. The 20 January and 28 October 2003 events both were preceded by interplanetary disturbances which resulted in Forbush decreases. That in January started around mid-day on 17 January and was around $-15 \%$ at Oulu, Finland by the beginning of 19 January. That preceding the 28 October 2003 event was around $-8 \%$ at Oulu. Thus, logically, if the structures in the inner heliosphere are keeping out the galactic cosmic rays at $\mathrm{GeV}$ energies, then they are likely to be preventing solar cosmic rays of similar energies from leaving the inner heliosphere. Hence the long decay, with a trend towards isotropy. This situation has been illustrated schematically by Simnett (2005a, his Fig. 7) following the original suggestion by Meyer et al. (1956). Events such as these are typically followed by another Forbush decrease when the CME from the initiating event passes over the Earth. Figure 10 compares the neutron monitor records from Oulu, Finland for the two events. If we compare the neutron monitor record with the magnetic field at ACE (Fig. 7) then the succession of Forbush decreases matches the time when the magnetic field strength is above $\sim 10 \mathrm{nT}$. For both events a depression in the galactic cosmic ray intensity of $\sim 10 \%$ is observed for several days prior to the GLE, via a succession of Forbush decreases.

\subsection{Electron spectrum}

We now turn to the electron differential energy spectrum. Simnett $(2005 a, b)$ has discussed the spectral signatures of different types of electron event and has suggested that the spectral index may be used to identify the source of the electrons, whether they be from the corona (in the absence of a flare), from a flare, or from a CME-driven shock. While his conclusions still need to be ratified, it is worth pointing out here that 
Table 1. Timing of various electromagnetic and particle emissions.

\begin{tabular}{lcccc}
\hline \hline Category & $\begin{array}{c}\text { Starting time } \\
1 \mathrm{AU}(\mathrm{UT})\end{array}$ & $\begin{array}{c}\text { Maximum time } \\
1 \mathrm{AU}(\mathrm{UT})\end{array}$ & $\begin{array}{c}\text { Starting time } \\
\text { Sun (ST) }\end{array}$ & $\begin{array}{c}\text { Maximum time } \\
\text { Sun (ST) }\end{array}$ \\
\hline $15.4 \mathrm{GHz}$ & $06: 35$ & $06: 44$ & $06: 27$ & $06: 36$ \\
SXR $(1-8 \mathrm{~A})$ & $06: 36$ & $07: 01$ & $06: 28$ & $06: 53$ \\
HXR(40 keV) & $06: 38$ & $06: 46$ & $06: 30$ & $06: 37$ \\
CME & $>06: 40$ & & $06: 32$ & $>06: 32$ \\
Flare (2B) & $06: 41$ & $06: 46$ & $06: 33$ & $06: 38$ \\
Gamma ray (4-7 MeV) & $06: 44$ & $06: 46$ & $06: 36$ & $06: 38$ \\
$14 \mathrm{MHz}(\mathrm{IP})$ & $06: 45$ & & $06: 37$ & \\
NM $(2 \mathrm{GeV})$ & $06: 49$ & $06: 53$ & $06: 39^{*}$ & $06: 43^{*}$ \\
E4 $(250 \mathrm{keV})$ & $06: 57$ & $07: 03$ & $06: 45^{*}$ & $06: 51^{*}$ \\
\hline
\end{tabular}

* Assuming scatter-free propagation of $1.1 \mathrm{AU}$ (protons $9.6 \mathrm{~m} \mathrm{@2} \mathrm{GeV;} \mathrm{electrons} 12.2 \mathrm{~m} @ 250 \mathrm{keV}$ ).

electron events with a spectral index $\gamma$ less than 2.5 are considered likely to be from a flare origin. Here the differential electron intensity $\mathrm{d} J / \mathrm{d} E \propto E^{-\gamma}$, where $E$ is the electron kinetic energy.

The spectral index of the peak sector is well-represented by $\gamma=1.45$ for the LEFS60 electrons and $\gamma=1.05$ for the deflected electrons. Thus the hard spectrum strongly suggests that we are witnessing directly-accelerated flare electrons. The differences in spectral index are probably due to ion contamination in the LEFS60 detector; thus we have more confidence to the spectrum from the deflected electrons.

Electrons from a CME-driven shock have a spectrum typically with $\gamma$ above 3 (Simnett 2005a). Therefore in this event, purely from spectral considerations, we would not expect the dominant electron component at ACE to have been accelerated by the CME-driven shock. The electron spectral index for $\mathrm{X}$-class X-ray flares where the intensity in the $38-53 \mathrm{keV}$ electrons exceeds $10^{6}$ electrons $\left(\mathrm{MeV} \mathrm{cm}^{2} \mathrm{sr} \mathrm{s}^{-1}\right.$ is between 1.25 and 2.5 for events detected at ACE from 1997-2004 (Simnett 2005c). The electrons from the 20 January event satisfy this criterion.

\section{Timing analysis}

The timing of the various electromagnetic and particle emissions is summarised in Table 1 . We are making use of remote sensing data from Earth (or near Earth) which have times given in UT, and in situ data, where the observed time is also in UT. However, for the particles we wish to derive the injection time at the Sun, which must have the propagation time subtracted. Therefore we define solar time (ST) as the Universal Time of an event at the Sun. Thus all the electromagnetic emissions have 8.3 min subtracted from the time observed at Earth, while the particle times are corrected by $L / v$. The onset times and times of maximum are given in Table 1 both in UT and ST.

The signatures of highly non-thermal electrons in the chromosphere are the hard X-ray burst and the microwave burst, for which we use the $15.4 \mathrm{GHz}$ data. There are no reliable proxies for relativistic protons in the chromosphere. However, at lower energies the neutron capture line $(2.223 \mathrm{MeV})$ is a proxy for protons $\sim 30 \mathrm{MeV}$, which is the threshold energy for breaking up ${ }^{4} \mathrm{He}$. Neutrons produced in this process are captured by ambient protons. Wang \& Ramaty (1974) showed that the neutron capture line was delayed from the production by $\sim 100 \mathrm{~s}$. The peak in the RHESSI channel which contains the neutron capture line (Fig. 2) is at 06:47 UT, 2 min after the peak in hard X-rays. The peak in the 4-7 MeV energy band, which contains the prompt gamma ray lines of $\mathrm{C}$ and $\mathrm{O}$, is at 06:46 UT (Dr G.H. Share, private communication). Thus the RHESSI data are consistent with contemporary acceleration of both energetic electrons and protons. If the acceleration process is rapid, production of relativistic protons would not be delayed significantly from that of the $30 \mathrm{MeV}$ protons.

The electromagnetic emissions only give information about the particles in or near the chromosphere; they do not give information about the escaping particles. The best information we have on these comes from the type III radio burst, which is produced by electron beams. Haggerty \& Roelof (2002) have noted that a typical electron beam seen by ACE is delayed from the interplanetary (IP) type III radio emission by around $10 \mathrm{~min}$. Their conclusions were similar to those of Krucker et al. (1999) for a similar study.

The onset of the electron event (Fig. 4) is at 06:57 UT which means they left the Sun at 06:44 ST. This is based on a velocity of $0.75 \mathrm{c}$ (electron kinetic energy of $262 \mathrm{keV}$ ) and a nominal path length of $1.1 \mathrm{AU}$. This is consistent with the typical delay (Haggerty \& Roelof 2002) from the onset of the $14 \mathrm{MHz}$ type III burst (Data courtesy of Dr M.L. Kaiser). The solar wind speed for the previous day was typically between 600 and $800 \mathrm{~km} \mathrm{~s}^{-1}$ (ACE data archive). The onset of the relativistic proton event was at 06:49 UT, which means they left the Sun no later than 06:39 ST. This is within a minute (later) of the maximum of the $0.8-7 \mathrm{MeV}$ gamma-ray burst shown in Fig. 1. As there is no reason to suppose the path length for the protons is different from that for the electrons, then the protons were injected some 5 min before the electrons.

Note that the major injection of the protons responsible for the ground level event at the South Pole is short lived and does not extend significantly beyond 06:54 UT, or 06:44 ST. However, it is clear from Fig. 2 (a) that the event continues to be anisotropic at least until after 08:20 UT, and (b) that the majority of neutron monitors, which typically have higher 


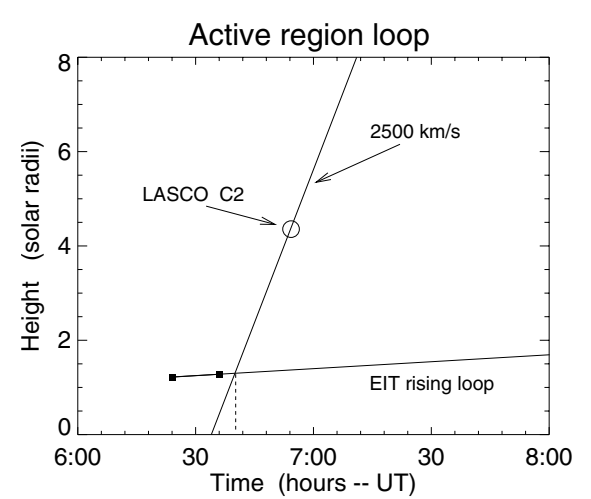

Fig. 11. The EIT rising loop and the $C 2$ image, put together as a suggested height-time plot. Note that the time at the Sun is $\sim 8$ min earlier than plotted. Also, the time markers are $6 \mathrm{~min}$.

threshold energies than the South Pole station, measure a similar intensity during the long decay. This would support the concept of a particle reservoir in the inner heliosphere, which provides the continued supply of protons at high energies; plus weak extended emission from the solar direction at the lower energies responsible for the additional South Pole response.

We now examine the coronal images to discover what was happening in the corona at the injection times of the relativistic particles. Unfortunately the cadence of the EIT and LASCO observations is an order of magnitude longer than the accuracy we are seeking to achieve. Therefore we must turn to a more subtle argument. We have shown in Fig. 6 that the image from the LASCO C2 coronagraph appears to show the leading edge of the CME attributed to the evolution of the rising loop seen in EIT, at around $4.34 R_{\odot}$. We shall call this the $\mathrm{C} 2$ point. If we take the projected speed to be $2500 \mathrm{~km} \mathrm{~s}^{-1}$, which is around the upper limit of CMEs observed by LASCO, then we may draw a line corresponding to this speed through the data point. This is shown in Fig. 11. The position angle of the measured C2 point is $309^{\circ}$. Position angle is measured anticlockwise from the solar north pole. We may now plot the positions of the top of the loop (leading edge) seen in Fig. 5. The intersection of the line through these points with the $2500 \mathrm{~km} \mathrm{~s}^{-1}$ line through the $\mathrm{C} 2$ point gives the time of the onset, assuming that the structure seen in the C2 image is actually the eruption of the loops seen by EIT. This time is 06:40 UT, or 06:32 ST. Note that the onset time calculated here is the latest possible CME onset time, as we have extrapolated back from the $\mathrm{C} 2$ point with the highest likely CME speed assuming instantaneous acceleration to that speed.

Taking all these data into consideration, we have the gamma-ray emission starting no later than 06:36 ST, the NM protons starting no later than 06:39 ST, and the CME launch time at or before 06:32 ST. However, the maximum of the NM protons is no later than 06:43 ST, while the gamma-ray maximum is at 06:38 ST. If the energetic protons are accelerated by the CME-driven shock, then there are two important questions: (1) why doesn't the gamma-ray emission continue to increase beyond 06:38 ST? and (2) why does the acceleration of the relativistic protons cease when the CME is at a few $R_{\odot}$ ? If the CME-driven shock is doing all the acceleration, then there

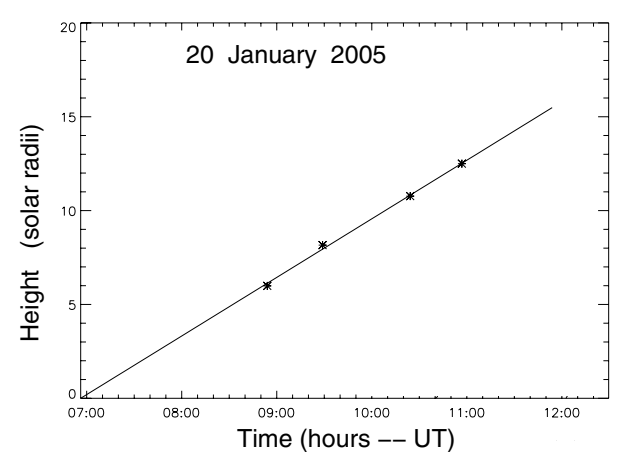

Fig. 12. The height-time plot ot the leading edge of the halo CME observed by LASCO following the major flare on 20 January.

is an additional question: (3) why isn't the near-relativistic electron injection at the same time as the protons?

We believe a solution that provides a satisfactory answer to these questions is that all the relativistic particle acceleration is at the flare site, and that it is effectively over by the time of the maximum of the $15.4 \mathrm{GHz}$ radio burst, the maximum of the HXR burst, and the maximum of the gamma ray burst. We suggest that the injection of the relativistic protons precedes that of the electrons because the turbulence or waves associated with the CME-driven shock traps the low rigidity electrons much more effectively than the protons. Hence release of the electrons is delayed somewhat, for this event by around $5 \mathrm{~min}$. Other events (Kahler et al. 2003) will have differing delays.

There may well be electrons accelerated by the CME. However, if there is a major flare component, as in the 20 January event, then emission into the interplanetary medium is dominated by the flare component. The energy spectrum is consistent with this. In the absence of a major flare, as in the impulsive beam electron events studied by Haggerty \& Roelof (2002) the CME source may dominate, with a corresponding spectral signature (Simnett 2005a,b).

\subsection{Additional considerations}

There was another CME visible in the LASCO C3 coronagraph which was bright enough to be detected despite the charged particle contamination. It first became clearly visible some two hours after the flare onset, when the particle contamination was beginning to die away. This event had a wide angular extent, which could have encompassed the solar disc as a halo. Figure 12 shows the height-time plot for the leading edge of the "halo" measured at a position angle of $109^{\circ}$, projected onto the plane of the sky. The projected speed is $604 \mathrm{~km} \mathrm{~s}^{-1}$. The important point from Fig. 12 is that this CME projected back to a nominal $1 R_{\odot}$ around 07:15 UT $\pm 5 \mathrm{~min}$, well after the flare and therefore could not be responsible for accelerating the relativistic protons. However, this indicates that the magnetic fields in the corona continued to evolve rapidly following the main event. As this CME is also probably heading directly towards the Earth, it is most likely responsible for the shock that hit the ACE spacecraft at 16:47 UT on 21 January, with a proton speed of over $940 \mathrm{~km} \mathrm{~s}^{-1}$ (Courtesy of the ACE data center). If this is true then the average speed is $\sim 1225 \mathrm{~km} \mathrm{~s}^{-1}$. 
This would then be consistent with a very fast CME near the Sun, yet there is no evidence for any additional proton acceleration, which supports our conclusion that the protons were accelerated in the flare. The magnetic field enhancement at the shock may be seen in Fig. 7.

\section{Conclusions}

The motivation for this work has been to provide constraints on the energy budget of solar flares. The hard X-ray observations provide information on the timing and the energy in the non-thermal electrons, which is normally interpreted according to the thick-target model originally postulated by Brown (1971). The energy in the flare ions (which are predominantly protons) is more uncertain, as it is plausible that the bulk of these may not actually come from the flare itself, but rather from the CME. The event we have discussed here provides the finest constraints on the timing of the relativistic proton acceleration that we have from any event studied to this date.

The primary evidence is the very fast rise of the $\mathrm{GeV}$ proton intensity detected by ground-level neutron monitors. An equally rapid decay is also a crucial point. We have shown that the near relativistic electron injection at the Sun is delayed by $\sim 6$ min from that of the $\mathrm{GeV}$ protons.

The conclusion we reach is that the protons responsible for the ground level neutron monitor event and those electrons detected by ACE/EPAM with a very hard spectral index were accelerated in a process or processes directly related to the solar flare, and were not accelerated by the CME. However, the $\mathrm{CME}$ was probably responsible for delaying the release of the flare electrons (from the release of the protons) by around $5 \mathrm{~min}$ onto magnetic field lines connected directly to the ACE spacecraft. The timing of the various emissions, the short duration of the relativistic proton event, and the energy spectrum of the detected electrons are the most critical pieces of evidence for this conclusion.

Finally, we point out that events such as 20 January, which are magnetically well-connected to the Earth, give effects at the Earth so quickly and with little warning, that they are particularly dangerous to astronauts and to the well being of many orbiting spacecraft.

Acknowledgements. The author wishes to thank Dr E.C. Roelof for his contributions to this paper. Thanks are due to colleagues on the ACE and Ulysses teams, and especially Dr S.J. Tappin, for their help in making the data available and in a useable form. Starlink computing facilities were used for the detailed analysis. We thank Prof John W. Bieber, Bartol Research Institute, University of Delaware and Dr C. Lopate, University of New Hampshire, for the use of the neutron monitor data. Neutron monitors of the Bartol Research Institute are supported by NSF grant ATM-0000315. The RHESSI data are kindly made available by the RHESSI consortium, with special thanks to Dr G.H. Share. The manuscript has been considerably improved following helpful comments by the referee.

\section{References}

Bieber, J. W., Dröge, W., Evenson, P. A., et al. 2002, ApJ, 567, 622

Bieber, J. W., Evenson, P., Dröge, W., et al. 2004, ApJ, 601, L103

Bieber, J. W., Clem, J., Evenson, P., et al. 2005, Geophys. Res. Lett., 32, L03S02

Brown, J. C. 1971, Sol. Phys., 18, 450

Brueckner, G. E, Howard, R. A., Koomen, M. J., et al. 1995, Sol. Phys., 162, 357

Cliver, E. W., Kahler, S. W., Shea, M. A., \& Smart, D. F. 1982, ApJ, 260,362

Debrunner, H., Lockwood, J. A., \& Ryan, J. M. 1993, ApJ, 409, 822

Delaboudinière, J.-P., et al. 1995, Sol. Phys., 162, 357

Forrest, D. J., \& Chupp, E. L. 1983, Nature, 305, 291

Gold, R. E., Krimigis, S. M., Hawkins, S. E., et al. 1998, Space Sci. Rev., 86, 541

Haggerty, D. K., \& Roelof, E. C. 2002, ApJ, 579, 841

Krucker, S., Larson, D. E., Lin, R. P., \& Thompson, B. J. 1999, ApJ, 519,864

Lin, R. P., Dennis, B. R., Hurford, G. J., et al. 2002, Sol. Phys., 210, 3

Kahler, S. W. 1994, ApJ, 428, 837

Kahler, S. W., Simnett, G. M., \& Reiner, M. J. 2003, Proc. 28th Int. Cosmic Ray Conf., 6, 3415

Meyer, P., Parker, E. N., \& Simpson, J. A. 1956, Phys. Rev., 104, 768

Ramaty, R. 1986, Physics of the Sun, II, ed. P. A. Sturrock (Dordrecht: Reidel) 291

Roelof, E. C., Gold, R. E., Simnett, G. M., et al. 1992, Geophys. Res. Lett., 19, 1243

Shea, M. A., Smart, D. F., \& Pyle, K. R. 1991, Geophys. Res. Lett., 18,1655

Simnett, G. M. 2005a, J. Geophys. Res., in press

Simnett, G. M. 2005b, Sol. Phys., in press

Simnett, G. M. 2005c, Sol. Phys., submitted

Simnett, G. M. 2003, Proc. ISCS 2003 Symp., Solar Variability as an input to the Earth's Environment, ESA SP-535, 613

Smith, C. W., L'Heureux, J., Ness, N. F., et al. 1998, Space Sci. Rev., 86,613

Wang, H. T., \& Ramaty, R. 1974, Sol. Phys., 36, 129 